Hautarzt 2014 - 65:280-281

DOI 10.1007/s00105-013-2701-3

Online publiziert: 11. April 2014

๑) Springer-Verlag Berlin Heidelberg 2014

C. Löser ${ }^{1}$ E. Haneke 2, 3, 4, 5

${ }^{1}$ Hautklinik, Hauttumorzentrum, Klinikum der Stadt Ludwigshafen am Rhein gGmbH, Ludwigshafen

2 Praxis für Dermatologie Dermaticum, Freiburg

${ }^{3}$ Dermatologische Klinik, Inselspital, Univ. Bern

${ }^{4}$ Centro de Dermatología Epidermis, Instituto CUF, Porto

${ }^{5}$ Kliniek voor Huidziekten, Academisch Ziekenhuis, Univ Gent

\title{
Klein, aber fein - Dermatologie, Dermatochirurgie und Nagelerkrankungen
}

„Klein, aber fein“ lautet das Motto der diesjährigen Strategiesitzung der Deutschen Gesellschaft für Dermatochirurgie (DGDC e.V.) vom 13. bis 14.06.2014 in Marburg. Ursprünglich wurden diese Tagungen zur Diskussion von fachpolitischen Strategien und der organisatorischen Justierung der Gesellschaft im Spannungsfeld zwischen ästhetischer Dermatologie und spezieller Dermatochirurgie eingeführt. Inzwischen haben sich die Strategiesitzungen, die alternierend zum großen Kongress der Gesellschaft abgehalten werden, zu wichtigen Fortbildungsveranstaltungen mit praktischem Schwerpunkt entwickelt. Das Team um den Tagungsleiter PD Dr. Wolfgang Pfützner in Marburg macht „Nägel mit Köpfen“ und hat ein Angebot mit hochinteressanten Kursen zusammengestellt. Es reicht von praktischen Kursen an Kadavern, Schweinepräparaten oder Bildschirmen über ein Angebot für Fachpersonal bis hin zu freien Vorträgen mit einem besonderen Augenmerk auf den kollegialen fachlichen Austausch. Als einen der Tagungshöhepunkte gibt es mit 2 Master-Lectures auch einen Einblick in die Möglichkeiten der Lymphchirurgie klein und fein. Und sollte Ihnen das Thema schon länger „auf den Nägeln brennen“ ... in Marburg bietet sich wieder Gelegenheit für die aktive Teilnahme an einem Nagelchirurgiekurs am Leichenpräparat.

„Klein, aber fein“ passt nicht nur zur DGDC-Tagung, sondern auch zu uns Dermatologen an sich. Als vergleichsweise überschaubare Gruppe innerhalb der Ärzteschaft verstehen wir es, auch mit minimalen und - wenn immer möglich schonenden Maßnahmen maximale Verbesserungen für den Patienten zu erzielen. Diese Einschätzung lässt sich perfekt auf die Dermatochirurgie mit den Fachbereichen Ästhetik, Phlebochirurgie, Laser und spezielle Dermatochirurgie übertragen. Nur ein gezieltes und schonendes Arbeiten wird in diesen Bereichen den heutigen Anforderungen an Funktionalität und Ästhetik gerecht.

Dermatologen können auch mit minimalen und schonenden Maßnahmen maximale Verbesserungen für den Patienten zu erzielen: Klein, aber fein.

„Klein, aber fein“ kann auch das Leitthema dieses Heftes überschrieben werden. Einige anerkannte Kapazitäten auf dem Gebiet der Diagnostik und Therapie von Nagelerkrankungen haben Beiträge beigesteuert, die Einblick in diese vermeintlich kleine, aber wichtige Materie vermitteln. Zum Einstieg erfahren wir Wissenswertes zur Biologie, Anatomie und Physiologie des Nagelorgans (Beitrag Haneke). Der Artikel über die Vielfalt der entzündlichen und neoplastischen Veränderungen gibt einen guten Eindruck von der Materie und macht Lust auf das intensivere Studium weiterführender Literatur (Beitrag Lutz et al.). Die Dermatoskopie des Nagels erlaubt eine nichtinvasive, einfach durchzuführende Diagnostik. Der Beitrag dazu gibt praktische Tipps zur Durchführung der Dermatoskopie an der harten, konvexen Nageloberfläche und fasziniert durch 
beeindruckendes Bildmaterial (Beitrag Haenssle et al.).

Die schonende und rechtzeitige $\mathrm{Be}$ handlung von Neoplasien der Nägel fordert den Operateur (Beitrag Haneke) und stellt ihn bei Kindern vor zusätzliche Herausforderungen (Beitrag Löser). Eine Herausforderung ist auch die Abklärung einer Pigmentierung des Nagels, die als „dunkler Nagel“ imponieren kann. Der entsprechende Beitrag liefert nicht nur Hilfestellungen zum praktischen Vorgehen, sondern richtet darüber hinaus einen Lichtstrahl auf die vielfältige Welt der Mykologie (Beitrag Löser et al.). Die Vielfalt der Infektionsproblematik des Nagelorgans als besonders exponierte Struktur des Körpers erfährt ebenfalls eine intensive Betrachtung (Beitrag Nenoff et al).

Zusammenfassend möchten wir allen Lesern die Teilnahme an der DGDC-Strategiesitzung in Marburg als ausgezeichnete Möglichkeit für Weiterbildung und Austausch empfehlen. Mögen darüber hinaus die Beiträge in diesem Heft nicht nur Ansporn zur Auseinandersetzung mit Nagelerkrankungen geben, sondern auch Anstoß zu gezielter Diagnostik sein und zum Behandlungserfolg beitragen.

Dr. Christoph Löser

Prof. Dr. Eckart Haneke

\section{Korrespondenzadressen}

\section{Dr. C. Löser}

Hautklinik, Hauttumorzentrum, Klinikum der

Stadt Ludwigshafen am Rhein gGmbH

Bremserstr. 79, 67063 Ludwigshafen

loeserc@klilu.de

\section{Prof. Dr. E. Haneke}

Praxis für Dermatologie Dermaticum Kaiser-Joseph-Str 262, 79098 Freiburg haneke@gmx.net

Interessenkonflikt. C. Löser und E. Haneke geben an, dass kein Interessenkonflikt besteht.

\section{Möchten Sie einen Beitrag für „Der Hautarzt” einreichen?}

\author{
Wir freuen uns, dass Sie unsere Zeit- \\ schrift Der Hautarzt mitgestalten \\ möchten. Um Ihnen bei der Manus- \\ kripterstellung behilflich zu sein, ha- \\ ben wir für unsere Autoren Hinweise \\ zusammengestellt, die Sie im Internet \\ finden unter www.DerHautarzt.de \\ (Für Autoren). \\ Bitte senden Sie Ihren fertigen Beitrag \\ an:
}

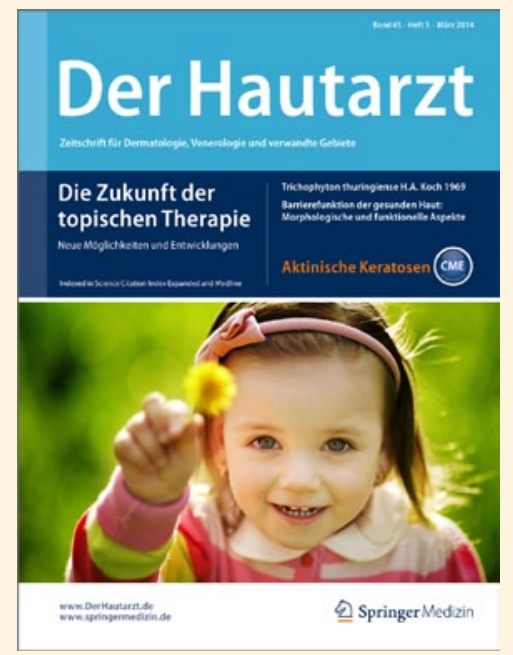

Originalien/Kasuistiken:

Prof. Dr. Alexander Kapp

Klinik für Dermatologie, Allergologie und Venerologie,

Medizinische Hochschule Hannover, OE 6600,

Carl-Neuberg-Str. 1, 30625 Hannover

derma@mh-hannover.de

Übersichten/ Wie lautet Ihre Diagnose?

Prof. Dr. Thomas Ruzicka

Klinik und Poliklinik für Dermatologie,

LMU München

Anfragen an:

Prof. Dr. Daniela Bruch-Gerharz

Hautklinik des Universitätsklinikums

Düsseldorf, Moorenstr. 5,

40225 Düsseldorf

Tel: +49-211-81-18328

Fax: +49-211-81-04905

s.gehrke@med.uni-duesseldorf.de

\author{
Weiterbildung . \\ Zertifizierte Fortbildung: \\ Anfragen an:
}

Prof. Dr. Michael Meurer

Klinik und Poliklinik für Dermatologie an der Universitätsklinik Carl Gustav Carus,

TU Dresden, Fetscherstr. 74,

01307 Dresden

meurer@rcs.urz.tu-dresden.de

Prof. Dr. Sonja Ständer

Klinik und Poliklinik für Hautkrankheiten,

Universitätsklinikum Münster

Von-Esmarch-Str. 58

48149 Münster

sonja.staender@uni-muenster.de

Prof. Dr. Rolf-Markus Szeimies

Klinik für Haut-, Allergie-, Venen- und

Umwelterkrankungen,

Knappschaftskrankenhaus Recklinghausen,

Klinikum Vest $\mathrm{GmbH}$,

Dorstener Str. 151,

45657 Recklinghausen

dermatologie@kk-recklinghausen.de

In der Diskussion:

Prof. Dr. Alexander Kapp/

Prof. Dr. Thomas Werfel

Klinik für Dermatologie, Allergologie und Venerologie,

Medizinische Hochschule Hannover, OE 6600,

Carl-Neuberg-Str. 1, 30625 Hannover derma@mh-hannover.de

\section{Leserforum:}

Prof. Dr. Hans F. Merk

Klinik für Dermatologie und Allergologie, Universitätsklinikum der RWTH, Pauwelsstraße 30, 52057 Aachen hans.merk@post.rwth-aachen.de 